\title{
La docencia online y presencial de la Historia en los nuevos planes de Comunicación
}

\author{
Fátima GIL GASCÓN \\ Universidad de Burgos \\ fatimagg@ubu.es \\ Javier MATEOS-PÉREZ \\ Universidad de Chile \\ javiermateos7@yahoo.es
}

Recibido: $17 / 10 / 2012$

Aceptado: 23/01/2013

\begin{abstract}
Resumen
El Espacio Europeo de Educación Superior coloca al alumno en el centro del proceso de enseñanzaaprendizaje. Esta permuta del paradigma educativo viene ligado a un cambio metodológico por parte del docente, con el fin de que potencie el papel activo del estudiante, su iniciativa y su pensamiento crítico. En este nuevo contexto, la enseñanza online, aplicando las Tecnologías de la Información y de la Comunicación, juega un papel clave, ofreciendo nuevos contextos y posibilidades para el desarrollo de estas competencias. Este investigación examina la aplicación de estas nuevas herramientas y da cuenta de cómo la universidad online amplía estas posibilidades. En especial, este estudio se centra en el análisis de la impartición online y presencial de la asignatura de Historia en los nuevos planes de la disciplina de la Comunicación.
\end{abstract}

Palabras clave: nuevas tecnologías, Espacio Europeo de Educación Superior, enseñanza online, Historia, Comunicación.

\section{OnLine and OnSite History Teaching in New Communication Plans}

\begin{abstract}
The European Higher Education Area places the student at the center of the teaching-learning process. This paradigm swap is accompanied by a methodological change by the teaching staff, with the end goal being an active role by the student where he or she takes the initiative and thinks critically. In this new format of online teaching, applying Information and Communication Technologies plays a key role in offering new ways and possibilities to develop these competencies. This investigation examines the application of new tools and shows how the online university amplifies these possibilities. Particularly, this study concentrates on the analysis of online teaching and on-site learning for the subject of History within the new communication plans
\end{abstract}

Keywords: New Technology, European Higher Education Area, Online Teaching, History, Communication

\section{Referencia normalizada}

GIL GASCÓN, Fátima y MATEOS-PÉREZ, Javier (2013): "La docencia online y presencial de la Historia en los nuevos planes de Comunicación”. Estudios sobre el mensaje periodístico. Vol. 19. Núm. especial abril, págs.: 803-811. Madrid, Servicio de Publicaciones de la Universidad Complutense.

Sumario: 1. Introducción, fuentes, estado de la cuestión. 2. Metodología. La aplicación de la asignatura de Historia en los planes EEES. 3. Desarrollo. La enseñanza de Historia en la Universidad presencial y virtual; 3.1. La Universidad Complutense de Madrid; 3.2. La Universidad Internacional de La Rioja. 4. Conclusión. La apuesta por los nuevos planes educativos. 5. Referencias bibliográficas.

\section{Introducción, fuentes, estado de la cuestión}

El Espacio Europeo de Educación Superior (EEES) se implantó en 1999 tras la firma de la conocida como declaración Bolonia, por la que los ministros de educación de los 
países miembros de la Unión Europea y los que aquellos países próximos a su adhesión (Declaración de Bolonia, 1999, web), se comprometieron a implantar un modelo universitario común con unas competencias que impulsan un aprendizaje más activo por parte del alumno (Calderón et al., 2008, 237).

Según las directrices adoptadas en esta y otras declaraciones, los estudios universitarios de los países de la unión debían consolidar el proceso de integración, y con él, el de creación de un Espacio europeo común en el año 2010.

Las modificaciones que el nuevo sistema universitario introducía en la educación superior eran sustanciales. En general y, según estudios realizados, la mayor parte de las universidades europeas han tenido (y tienen) dificultades más o menos semejantes. Las más relevantes son:

1. La organización de los estudios universitarios en ciclos. Ésta nueva reestructuración no implica una condensación de las materias en menos tiempo de estudio -el grado no es una licenciatura de menor duración- sino una nueva concepción del aprendizaje y la enseñanza.

2. El establecimiento de unos créditos ECTS que miden no sólo la asistencia a clase del estudiante sino, también, el trabajo que este desarrolla fuera del aula. Esta cuestión es especialmente interesante puesto que cambia completamente el sujeto a través del cual se cuantifica el trabajo. El estudiante deja de ser un sujeto pasivo, un receptor de información, para ser un sujeto activo en el proceso de aprendizaje.

3. El planteamiento práctico de los estudios. Los nuevos planes tiene como objetivo prioritario formar al estudiante en los conocimientos teóricos pero, sobre todo, los necesarios para su vida profesional. A nivel práctico, esto implica un planteamiento diferente de la enseñanza de debe ir más allá de las clases magistrales e involucrar a profesores y alumnos en la realización de diferentes trabajos y ejercicios que constituirán buena parte de la nota de los estudiantes.

En España, la implantación del plan Bolonia se está realizando, en la mayor parte de las universidades de forma progresiva.

Uno de los organizamos creados para controlar la implantación de esta nueva organización de la enseñanza superior fue la ANECA. Fundada en el año 2002, tiene como función adecuar las titulaciones existentes al nuevo marco de estudios, no sólo verificando la implantación de los nuevos grados sino, también, controlando que los principios del plan Bolonia sean llevados a cabo a través de auditorías.

Entre el año 2008 y el 2010 se realizado 2.464 informes definitivos de grados universitarios de los cuales 2.358 -un 96\%- consiguieron una evaluación favorable (ANECA, 2012, web). Los criterios en los que se basa la ANECA para la concesión de la evaluación positiva son muy concretos: descripción y definición del título que el estudiante va a recibir una vez termina sus estudios, justificación y utilidad/necesidad de la existencia del mismo, planteamiento de las competencias que el estudiante adquirirá con la titulación, explicación de los criterios de acceso y admisión de los alumnos, planificación de las materias en función de los principios establecidos por el plan Bolonia, planteamiento de los recursos tanto materiales como el capital humano 
con el que se va a contar para implantar los estudios, los resultados que se esperan conseguir, los criterios de calidad impuesto para controlar el buen funcionamiento del grado y, por último, un calendario en el que se especifique el tiempo cuando y de qué manera se va a implantar el nuevo modelo educativo.

Entre los años 2009 y 2010 comenzaron los primeros estudios de grado en la mayor parte de las universidades españolas. En la actualidad, muchas todavía mantienen grupos residuales de licenciatura.

\section{Metodología. La aplicación de la asignatura de Historia en los planes EEES}

En 1971, un decreto del ministerio de Educación y ciencia permitía la creación de las primeras facultades de Ciencias de la Información en la universidad Complutense y en la Autónoma de Barcelona. Se reconocía también oficialmente la facultad de Ciencias de la información de Navarra.

En la UCM, el plan de estudios definitivo que se mantuvo vigente hasta el año 1995 fue aprobado por una orden ministerial del 21 de octubre del año 1975 (Pérez Herrero, 2001, 3). Este plan separaba las ramas de Periodismo, Publicidad y Ciencias de la imagen visual y auditiva. En los tres casos se incluía una asignatura de Historia Contemporánea anual, incluyéndose otra asignatura de Historia en el tercer curso de Periodismo.

Las escuelas de periodismo habían ya incorporado, mucho antes de su transformación en estudios universitarios, la asignatura de Historia entendiendo la necesidad de una formación específica del futuro comunicador en esta área (Pérez Herrero, 2001, 202). Esta materia se consideraba, junto a la lengua española o la economía, una buena manera de dotar a los futuros comunicadores de una formación más o menos completa, que les permitiera profundizar en la información que iban a transmitir.

Los planes universitarios posteriores, tanto de la universidad complutense como del resto de las facultades de comunicación que fueron creándose a lo largo de los años ochenta y noventa, incorporaron también la enseñanza de la Historia como una asignatura obligatoria que se cursaba en los primeros cursos. Una asignatura que se entendía y presuponía básicamente teórica.

El presente estudio tiene como objetivo analizar de qué manera pueden adaptarse las asignatura de Historia en un contexto como el Espacio Europeo Superior de Educación en el que se pretende primar el aprendizaje práctico frente al teórico y en un carrera en la que esta este tipo de enseñanzas destinadas al desarrollo profesional son especialmente importantes.

Cómo ya se ha indicado, la mayor parte de los planes de estudio de las licenciaturas de Periodismo, comunicación audiovisual o publicidad tenían alguna asignatura de Historia de carácter obligatorio en los primeros años. Lo mismo sucede actualmente con los grados.

La adecuación de este tipo de disciplina a un contexto educativo como el propuesto en el plan Bolonia puede resultar compleja si se tiene en cuenta su carácter teórico. Para observar de qué manera es posible adaptar los estudios de la Historia a los grados de comunicación se ha procedido a la comparación de la enseñanza de esta disciplina en dos universidades muy distintas: la Universidad Complutense de Madrid, una veterano centro docente, de naturaleza pública y metodología presencial, y la Uni- 
versidad Internacional de La Rioja, una recién creada Universidad privada, cuya principal característica es que su docencia se imparte íntegramente online.

Ambos centros son muy diferentes tanto por su naturaleza, por su administración, por su metodología, así como por el tipo de alumno matriculado. La primera suele mantener un alumnado joven, de dieciocho a veintitantos años, cuya edad coincide con el ciclo académico. El alumno tipo carece de cargas familiares y ocasionalmente compatibiliza su trabajo académico con la actividad profesional a tiempo parcial. Los estudiantes matriculados en la segunda responden a tres perfiles: jóvenes trabajadores de unos veinticinco años, hombre y mujeres de treinta a cuarenta años con cargas familiares, profesionales del área de la comunicación de más de cuarenta años sin titulación.

Para establecer la comparación entre ambos tipos de enseñanza se ha procedido a analizar los siguientes parámetros:

a) Definición del centro.

b) Planteamiento de la metodología docente utilizada. Para ello se ha procedido tanto a la explicación de los métodos utilizados por el profesor como a las respuestas de una pequeña entrevista que aborda algunos puntos importantes:

-¿Cuántos alumnos suelen acudir a clase?

$-i$ Se muestran participativos los alumnos?

- ¿Se implican en la asignatura? Se entiende que hay una implicación en la misma cuando hay un marcado interés en la materia o cuando el alumno realiza actividades que no computan en la nota final.

-¿Es frecuente el contacto con sus alumnos? En este caso se contemplan tanto las tutorías como las preguntas a través del correo electrónico.

-¿Utiliza usted power point para sus explicaciones? ¿Lo deja a disposición de sus alumnos?

-¿Utiliza material audiovisual?

-¿Fomenta usted el aprendizaje crítico a través de discusiones o foros de debate?

c) Organización de la actividad docente y del trabajo de los alumnos. Cuantificación de los resultados académicos (número de aprobados y suspensos)

\section{Desarrollo. La enseñanza de Historia en la Universidad presencial y virtual 3.1. La Universidad Complutense de Madrid}

La Universidad Complutense de Madrid fue fundada en 1499 en la localidad de Alcalá de Henares. En el año 1836, gracias a una Real Orden dictada por Isabel II a través de la reina regente, decretó su traslado a Madrid, lo que sirvió primero para la fundación de la Nueva Universidad de Madrid, más tarde Universidad Literaria de Madrid, y poco después Universidad Central. Finalmente, más de un siglo después de empezar a funcionar, adoptó el nombre de Universidad Complutense de Madrid. En 1971 se regularon los estudios de periodismo y comunicación social en España y posteriormente se crearon las primeras facultades de Ciencias de la Información de nuestro país, entre ellas la Universidad Complutense, cuyas clases comenzaron a impartirse a partir de 1972.

El programa docente del Plan de Estudios de la Universidad Complutense de 1995 establecía dos asignaturas troncales y obligatorias en el primer ciclo, radicadas en el ámbito de la Historia. Historia Universal Contemporánea, asignatura troncal de seis 
créditos, impartida durante el primer curso; e Historia de España Contemporánea, asignatura obligatoria de cuatro créditos, impartida durante el tercer curso.

La naturaleza de la universidad pública y presencial plantea ciertos rasgos distintivos. Las clases presenciales se realizan en un aula única donde se van sucediendo las distintas sesiones tanto teóricas como prácticas.

Además, en estas clases se pretende una comunicación constante con el alumno, espoleando su participación continua con la mezcla de preguntas, debates y comentarios críticos al rebufo de lo expuesto en el apartado teórico. Por otra parte, profesor y alumno también interaccionan a través de las tutorías, previamente concertadas y en horario estanco, así como mantienen una comunicación fluida mediante el cauce del correo electrónico. Por último, mencionar la herramienta online del Campus Virtual, donde el profesor sitúa materiales de apoyo para las clases, así como enlaces a direcciones de interés para la asignatura y distintas comunicaciones para el grupo -bibliografía, actividades, calificaciones, etcétera-.

El sistema de calificación establecido por la Universidad Complutense deja al profesor libertad para escoger el método de evaluación. En este caso, se ha optado por basar la calificación final de la asignatura en función de tres parámetros: asistencia y participación en clase ( 20 por ciento), trabajo crítico ( 20 por ciento) y examen escrito (60 por ciento). A pesar de que la Facultad no destaca por el hecho de conceder especial relevancia a los conocimientos prácticos, en esta asignatura se pretende revertir esta situación con la vista puesta en los nuevos planes educativos.

El alumno accede a los contenidos teóricos gracias a las mencionadas sesiones presenciales, que se presentan al alumno como seminario temático, al Campus Virtual ya mencionado y a un manual de referencia para seguir la asignatura. El conocimiento del alumno sobre la teoría requerida será evaluado en un examen presencial datado en las convocatorias oficiales.

En cuanto al contenido práctico, habría que dividirlo en dos secciones: prácticas o trabajo en clase, y trabajo en casa mediante lecturas y análisis de fuentes primarias artículos, novelas, material audiovisual, ensayos-. Por último mencionar que el grueso de actividades realizadas por los alumnos deben de exponerse en clase, aunque debido al número de alumnos asistentes, se busca una selección de los mismos que sirvan de ejemplo extrapolable al resto de alumnos.

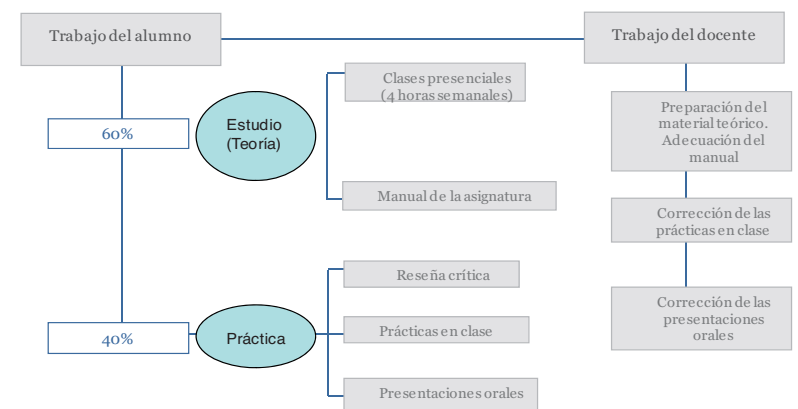

Gráfico 1: elaboración propia a partir de la información del programa académico de la asignatura Historia del mundo contemporáneo 
La asignatura de Historia del mundo contemporánea es compartida por dos profesores, siendo uno de ellos doctor en periodismo y el otro doctor en historia. Las herramientas utilizadas por los docentes son similares: explicaciones en clase teóricas, aderezadas con presentaciones concretas, análisis de material audiovisual, textos periodísticos o audios.

Los resultados de los alumnos de Historia del mundo contemporáneo arrojan el siguiente balance cuantitativo:

\begin{tabular}{|c|c|c|c|c|}
\hline Año & $\begin{array}{c}\text { \% actividades en- } \\
\text { tregadas }\end{array}$ & $\begin{array}{c}\text { \% actividades } \\
\text { aprobadas }\end{array}$ & $\begin{array}{c}\text { Alumnos aproba- } \\
\text { dos convocatoria } \\
\text { ordinaria }\end{array}$ & $\begin{array}{c}\text { Alumnos aproba- } \\
\text { dos convocatoria } \\
\text { extraordinaria }\end{array}$ \\
\hline $2008 / 2009$ & $70 \%$ & $64,9 \%$ & 60 & 10 \\
\hline $2008 / 2009$ & $55 \%$ & $42,15 \%$ & 53 & 6 \\
\hline
\end{tabular}

(Elaboración propia según datos proporcionados por la universidad)

Aproximadamente un 68,5 por ciento de los alumnos matriculados en el curso -es necesario matizar que se trata del análisis de sólo dos grupos de ocho en total- aprueba la asignatura entre ambas convocatorias. Un porcentaje con mínimas diferencias con respecto al porcentaje de alumnos que entregan las actividades durante el curso.

\subsection{La Universidad Internacional de La Rioja}

La Universidad Internacional de la Rioja es una universidad online creada en el año 2009 dentro del marco del Espacio europeo de educación superior. Uno de los primeros grados que comenzaron a impartirse fue el de Comunicación en la faculta de Empresa y comunicación, verificado por la ANECA en julio del $2009^{1}$.

El programa docente establecía que en el primer año se cursaban dos asignaturas de Historia: Historia del mundo del siglo XX, una asignatura básica de 6 créditos del primer cuatrimestre e Historia de España del siglo XX, también de carácter básico y de 6 créditos, pero impartida en el segundo cuatrimestre.

En el año 2012, esta universidad comienza el cuarto curso del grado manteniendo un importante crecimiento de alumnos.

La naturaleza online de la universidad plantea ciertas peculiaridades. Las clases presenciales se realizan a través de una cámara web que permite a los alumnos acceder a la misma desde cualquier lugar. Éstas son grabadas pudiendo acceder el estudiante a ellas tantas veces como necesite. Además de estas sesiones en las que el alumno interactúa con el profesor a través de un chat, el alumno tiene a su disposición otro tipo de clases grabadas, que actuarían como seminarios, de unos diez minutos de duración en las que el profesor explica una cuestión concreta o realiza una entrevista a algún experto sobre un tema determinado.

Además de en las clases presenciales, las relaciones entre alumnos y profesores se establecen a través de dos medios diferentes: un foro en el que el alumno indica las

${ }^{1}$ Memoria del título oficial de Grado en Comunicación. Disponible en http://www.unir.net /grado-comunicacion.aspx consultado el 12 de septiembre del 2012. 
dudas o planteamientos y el correo electrónico. El profesor no tiene un horario de tutorías sino que se compromete a contestar las dudas planteadas por el estudiante, a través del correo, tan pronto como le sea posible.

El sistema de calificación establecido por esta universidad hace un especial hincapié en la importancia de los conocimientos prácticos. El alumno accede en su campus virtual al contenido teórico de la asignatura, preparado generalmente por el profesor, así como a diferentes materiales complementarios -lecturas, películas, glosario de términos- que le permiten, junto a la clase semanal que imparte el docente alcanzar un aprendizaje teórico de la materia. Éste será evaluado en un examen final presencial que constituye el $60 \%$ de la nota.

Además de esto el alumno debe realizar una serie de actividades para conseguir el $40 \%$ restante de la nota y que constituyen la parte no-teórica de la materia. Estos ejercicios suelen ser de tres tipos: comentarios de texto de libros o de artículos, actividades que implican una cierta investigación y foros de debate en los que se plantean determinadas cuestiones que el alumno debe comentar justificando sus comentarios.



Gráfico 2: elaboración propia según información de las guías docentes de las asignaturas Historia del mundo del siglo XX e Historia de España del siglo XX.

Las asignaturas de Historia del mundo e Historia de España del siglo XX son impartidas por sendos profesores, ambos doctores en periodismo. Las herramientas utilizadas por los docentes son más o menos semejantes: principalmente un power point en el que se explicitan las cuestiones más importantes del tema explicado, y, cuando es posible, fuentes primarias (material audiovisual, textos, periódicos, audios...).

Los resultados de los alumnos son semejantes en ambas asignaturas. Los alumnos de Historia del mundo del siglo XX pueden realizar, para alcanzar la máxima puntuación en el $40 \%$ correspondiente a la parte práctica, un total de 10 ejercicios.

\begin{tabular}{|c|c|c|c|c|}
\hline Año & $\begin{array}{c}\% \text { actividades en- } \\
\text { tregadas }\end{array}$ & $\begin{array}{c}\% \text { actividades } \\
\text { aprobadas }\end{array}$ & $\begin{array}{c}\text { Alumnos aproba- } \\
\text { dos convocatoria } \\
\text { ordinaria }\end{array}$ & $\begin{array}{c}\text { Alumnos aproba- } \\
\text { dos convocatoria } \\
\text { extraordinaria }\end{array}$ \\
\hline $2009 / 2010$ & $36,59 \%$ & $34,15 \%$ & 16 & 3 \\
\hline $2010 / 2011$ & $57,5 \%$ & $54,57 \%$ & 36 & 18 \\
\hline $2011-2012$ & $52,2 \%$ & $49,9 \%$ & 60 & 10 \\
\hline
\end{tabular}


Los alumnos de Historia de España del siglo XX pueden realizar, para alcanzar la máxima puntuación en el $40 \%$ correspondiente a la parte práctica, un total de 10 ejercicios.

Aproximadamente un 50\% de los alumnos matriculados en el curso -evidentemente esto depende también del grupo analizado- aprueba la asignatura entre ambas convocatorias. Un porcentaje muy similar al que realizan las actividades propuestas y calificadas por el profesor a lo largo del cuatrimestre.

\section{Conclusión. La apuesta por los nuevos planes educativos}

La experiencia exploratoria que se ha llevado a cabo tiene una de serie de peculiaridades que conviene resaltar. En primer lugar, las asignaturas de Historia impartidas en la Universidad Complutense, a pesar de pertenecer al plan antiguo, se toman por parte de los profesores como una preparación para la convergencia con el Plan de Bolonia. Es decir, con estos grupos de alumnos, a pesar de ser cinco veces más numerosos que los calculados para el EEES, ya se pretende trabajar con una dinámica cercana al nuevo plan educativo. A pesar de que los resultados son positivos, en cuanto al número de alumnos aprobados, al porcentaje de prácticas entregadas y a la valoración final de la asignatura por parte de los estudiantes, el trabajo del docente se incrementa de manera notable. Por otra parte, en el caso de las asignaturas de Historia impartidas en la Universidad Internacional de La Rioja, que ya se inician en el Plan de Bolonia, nos encontramos un problema similar, puesto que el número de alumnos también sobrepasan el mínimo recomendado y esto obliga al docente a invertir un mayor tiempo en la preparación de los contenidos, correcciones y evaluación de la asignatura.

La dinámica de enseñanza no varía de una Universidad a otra más que por la metodología presencial de la Universidad Complutense y por la utilización casi exclusiva de las nuevas tecnologías en el caso de la Universidad Internacional de La Rioja. Tanto en uno como en el otro centro docente se pretende pasar de un modelo de lecciones magistrales a otro que fomente un mayor intercambio entre profesor y alumno. Los estudiantes no se comportan de manera pasiva, puesto que deben trabajar más en casa y acudir a las clases para completar lo estudiado y debatir posibles temas. De este modo se fomenta la capacidad argumentativa de los estudiantes, quienes cuentan con la ayuda del profesorado para corregir y mejorar. Así, por ejemplo, los alumnos no escuchan al profesor hablar sobre el discurso de Churchill, sino que debatirán sobre sus diversos puntos de vista tras haberlo leído previamente en casa.

Por su parte, y unido a esto, el profesorado hace un uso cada vez mayor de las nuevas tecnologías en sus clases. En consecuencia aparecen nuevas sesiones con nuevos materiales que sirven para enriquecer la enseñanza haciéndola, no sólo más dinámica, sino también más completa para unos alumnos cada vez más entrenados en estas herramientas.

Con los créditos ECTS se supera el problema de que los créditos no correspondan de manera objetiva con las horas de trabajo, puesto que en el experimento se reconocen las horas no lectivas que los alumnos invierten en su aprendizaje. Además, en relación con el aprobado, éste se consigue más fácilmente porque se tiene en cuenta, no sólo la calificación del examen final, requisito único en el plan antiguo, sino todo el 
trabajo que se realice durante el curso. Así, los alumnos que trabajen en el día a día y que asistan regularmente a las clases, podrán terminar sus respectivos estudios en los años previstos y con unas mejores calificaciones.

Por todo ello, parecen existir numerosas razones que afirman que el Plan de Bolonia, si mantiene el número de alumnos recomendados para su implantación, es efectivo para los estudiantes y, por lo tanto, merece la pena acometerlo.

\section{Referencias bibliográficas}

ANECA (2011): "Programa Verifica". ANECA, disponible en: http://www.aneca.es /Programas/VERIFICA [Consultado: 17 de septiembre de 2012].

BENITO, Águeda Y CRUZ, Ana (2005): Nuevas claves para la docencia universitaria en el espacio europeo de educación Superior. Madrid, Narcea.

CALDERÓN PATIER, Carmen y ESCALERA IZQUIERDA, Gregorio (2008): La educación de la docencia ante el reto del espacio europeo de educación superior, en Educación XXI, núm. 11, UNED, 2008.

CAMACHO, Enriqueta; GARCÍA, Ma Paz; MASERO Inmaculada; VÁZQUEZ M ${ }^{\mathrm{a}}$ José; y ZAPATA Asunción (2007): "Mínimos que maximizan. Equivalencia entre un crédito europeo y el trabajo de un profesor". XV encuentro de ASEPUMA y III Encuentro internacional. 20, 21 de septiembre. Mallorca.

CEBRIÁN, Manuel (2003): La enseñanza virtual para la innovación universitaria. Madrid, Narcea.

DECLARACIÓN DE BOLONIA (1999): Declaración conjunta de los Ministros europeos de Educación reunidos en Bolonia el 19 de junio.

PÉREZ HERRERO, Julio César (2001): Lineas de investigación y aportaciones docentes de los teóricos españoles a la Tesorería general de la información (19711996). Tesis doctoral. Madrid, Universidad Complutense de Madrid.

RUÉ, Joan (2007): Enseñar en la Universidad. El EEES como reto para la educación superior. Madrid, Narcea.

\section{Fátima GIL GASCÓN}

Universidad de Burgos

Doctora en Periodismo por la Universidad Complutense de Madrid. Profesora ayudante doctora en el Grado de Comunicación de la Universidad de Burgos fatimagg@ubu.es

\section{Javier MATEOS-PÉREZ}

Universidad de Chile

Doctor en Periodismo por la Universidad Complutense de Madrid. Profesor Asistente de la Universidad de Chile, en el Instituto de la Comunicación e Imagen

javiermateos7@yahoo.es 